\title{
MINIMAL GRAPHS WITH DISCONTINUOUS BOUNDARY VALUES
}

\author{
ROBERT HUFF ${ }^{\bowtie}$ and JOHN MCCUAN
}

(Received 2 January 2007; accepted 3 September 2007)

Communicated by M. G. Cowling

\begin{abstract}
We construct global solutions of the minimal surface equation over certain smooth annular domains and over the domain exterior to certain smooth simple closed curves. Each resulting minimal graph has an isolated jump discontinuity on the inner boundary component which, at least in some cases, is shown to have nonvanishing curvature.
\end{abstract}

2000 Mathematics subject classification: primary 35J67; secondary 53A10.

Keywords and phrases: minimal surface equation, Weierstrass representation, extremal length, boundary regularity.

\section{Introduction}

The boundary value problem

$$
\operatorname{div}\left(\frac{D u}{\sqrt{1+|D u|^{2}}}\right)=0 \quad \text { on } \Omega,
$$

for the minimal surface equation on a smooth bounded domain $\Omega \subseteq \mathbb{R}^{2}$ is solvable for arbitrary continuous boundary values $u_{0}$ if and only if $\Omega$ is convex [JS68]. On the other hand, solutions to Equation (1) may be obtained by minimizing the functional

$$
\mathcal{F}[u]=\int_{\Omega} \sqrt{1+|D u|^{2}}+\int_{\partial \Omega}\left|u-u_{0}\right| d \mathcal{H}^{1} .
$$

A minimizer always exists in $C^{\infty}(\Omega) \cap W^{1,1}(\Omega)$ even if $\Omega$ is not convex and even if $u_{0}$ is only in $L^{1}(\partial \Omega)$; see [Giu84]. It follows that the trace of such a minimizer $u$ is always well defined, and it makes sense to ask for conditions under which the

(C) 2009 Australian Mathematical Society 1446-7887/2009 \$16.00 

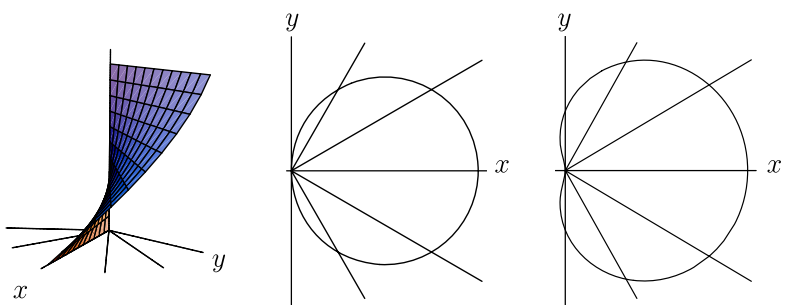

FIGURE 1. A helicoidal jump discontinuity.

boundary condition (2) will also hold. Many authors have addressed this and related questions [Mir71, Sim74, Sim82, Wil84, Wil86a, Wil86b, Wil87, ET99]. We mention, in particular, that if $x_{0} \in \partial \Omega$ is a point of positive curvature with respect to the normal pointing into $\Omega$ and $u_{0}$ is continuous at $x_{0}$, then

$$
\lim _{\Omega \ni x \rightarrow x_{0}} u(x)=u_{0}\left(x_{0}\right) .
$$

Furthermore, if the curvature of $\partial \Omega$ has a nondegenerate change of sign at $x_{0}, u_{0}$ is continuous at $x_{0}$ and $u_{0}$ is globally bounded, then (4) holds [Sim74, Wil86b]. In the case of negatively curved boundary and Lipschitz $u_{0}$, it is known that minimizers of (3) may differ in trace from $u_{0}$. Even in this situation, however, the trace of $u$ will still be Lipschitz.

It is also possible for minimizers of (3) to have discontinuous boundary values when $u_{0}$ is continuous, but this is only known to occur when the curvature of $\partial \Omega$ vanishes to first order. Of course, one may also consider the boundary value problem (1) and (2) or the minimization problem in (3) with specific discontinuous $u_{0}$, and presumably there are many solutions taking prescribed discontinuous boundary values. We are unaware, however, of any general result to this effect. One particularly interesting solution of this sort over a convex domain appears in [Fin63]. Also in the convex case, Lancaster's recent resolution of the Concus-Finn conjecture [Lan06] should provide many examples simply by taking capillary data with a jump discontinuity.

The possibility of discontinuities occurring locally on a portion of smooth boundary of any curvature is easily verified by cutting out a portion of a helicoid as follows. Let

$$
u(x, y)=\arg (x, y):= \begin{cases}\tan ^{-1}(y / x), & x>0, \\ \pm \frac{\pi}{2}-\tan ^{-1}(x / y), & \pm y>0, x \leq 0,\end{cases}
$$

for $(x, y)$ either in the ball $B_{r}(r, 0)$, for positive curvature $\kappa=1 / 4$, or in the domain bounded by the limaçon

$$
\gamma(t)=(r+b \cos t)(\cos t, \sin t)+(r-b, 0)
$$

where $0<b<r$ (see Figure 1). In fact, if we consider $0 \leq b<r$, we obtain both kinds of domains and the curvature at the origin (below the vertical axis of the helicoid) is 
found to be

$$
\kappa=\frac{r-2 b}{(r-b)^{2}} .
$$

It is also evident that the helicoid in this example may be scaled to give a jump discontinuity of arbitrary height. It is not possible, however, to modify this example in such a way that the discontinuity is located on the inner boundary of a doubly connected domain. A particularly elusive global question, as posed by John Urbas, turns out to be the following:

'Is it possible to find a minimal graph over a smooth annular domain of nonvanishing curvature with an isolated jump discontinuity occurring on the inner boundary component?'

We answer this question as the following result.

THEOREM 1. For each $\theta \in[0, \pi / 2)$, there exists an annulus $\mathcal{A}_{\theta}$, whose inner boundary component, except at possibly two points $a_{1}$ and $a_{2}$, has strictly negative curvature with respect to the inward pointing normal, and a minimal graph $M_{\theta}=$ $\operatorname{Graph}\left(u_{\theta}\right)$ over $\mathcal{A}_{\theta}$ such that the function $u_{\theta}$ has a finite jump discontinuity at $a_{1}$ and $a_{2}$. The curvature $k$ at the points $a_{1}$ and $a_{2}$ is as follows:

(i) if $0 \leq \theta<\pi / 4$, then $k\left(a_{1}\right)=k\left(a_{2}\right)=0$;

(ii) if $\theta=\pi / 4$, then $-\infty<k\left(a_{1}\right)=k\left(a_{2}\right)<0$;

(iii) if $\pi / 4<\theta<\pi / 2$, then $k\left(a_{1}\right)=k\left(a_{2}\right)=-\infty$.

It is also of interest to construct minimal graphs with inner boundary component as described above and extending over an entire exterior domain. A technical difficulty in this case is controlling the asymptotic behavior of the surface at infinity. Nevertheless, we have the following result.

THEOREM 2. For each $\theta \in[0, \pi / 2)$, there is an annular exterior domain with inner boundary as described in Theorem 1, which supports a minimal graph with jump discontinuities at two points.

It should be possible to obtain these 'entire' solutions as limits of the graphs in Theorem 1, but we have been unable to show that.

\section{Basic ingredients}

This section describes the tools we use to prove Theorems 1 and 2 .

2.1. The Weierstrass representation Given a domain $\Omega \subset \mathbb{C}$, the Weierstrass representation theorem says that any (orientation-preserving) conformal minimal immersion

$$
X=\left(X_{1}, X_{2}, X_{3}\right): \Omega \rightarrow \mathbb{R}^{3}
$$


can be expressed, up to translation, in terms of a meromorphic function $g$ and a holomorphic one-form $d h$ by the formula

$$
X(z)=\operatorname{Re} \int^{z}\left(\frac{1}{2}\left(g^{-1}-g\right) d h, \frac{i}{2}\left(g^{-1}+g\right) d h, d h\right),
$$

where $g$ is the stereographic projection of the Gauss map and

$$
d h=\left(\frac{\partial X_{3}}{\partial x}-i \frac{\partial X_{3}}{\partial y}\right) d z
$$

is called the complex height differential (note that $\operatorname{Re}(d h)=d X_{3}$ ). Conversely, the theorem states that if $g$ is a meromorphic function and $d h$ a holomorphic one-form on $\Omega$ such that $d h$ has a zero of order $n$ at $z$ if and only if $g$ has a zero or pole of order $n$ at $z$, then (5) gives an (orientation-preserving) conformal minimal immersion on $\Omega$ that is well defined provided that

$$
\operatorname{Re} \int_{\gamma}\left(\frac{1}{2}\left(g^{-1}-g\right) d h, \frac{i}{2}\left(g^{-1}+g\right) d h, d h\right)=0
$$

for every simple closed curve $\gamma \subset \Omega$. (Note that (6) is satisfied automatically if $\Omega$ is simply connected.) For a more extensive introduction to the Weierstrass representation see [HK97].

2.2. Determining $\boldsymbol{d} \boldsymbol{h}$ via the second fundamental form For a minimal surface given by Weierstrass data $g$ and $d h$,

$$
\frac{d g(v) d h(v)}{g}=I I(v, v)-i I I\left(v, \operatorname{Rot}_{\pi / 2}(v)\right),
$$

for a tangent vector $v$, where $I I$ is the second fundamental form on the surface. In particular, from (7) it follows that

$$
\gamma \text { is a principal curve } \Leftrightarrow \frac{d g(\dot{\gamma}) d h(\dot{\gamma})}{g} \in \mathbb{R}
$$

and

$$
\gamma \text { is an asymptotic curve } \Leftrightarrow \frac{d g(\dot{\gamma}) d h(\dot{\gamma})}{g} \in i \mathbb{R} .
$$

Thus, we see from (8) and (9) that the function $\zeta$ given by

$$
\zeta(z)=\int^{z} \sqrt{\frac{d g d h}{g}}
$$

maps principal curves into vertical or horizontal lines and asymptotic curves into lines in one of the directions $e^{ \pm i \pi / 4}$. The map $\zeta$ is called the developing map of the oneform $\sqrt{d g d h / g}$. It is a local isometry between the minimal surface equipped with the conformal cone metric $|d g d h / g|$ and $\mathbb{R}^{2}$ equipped with the Euclidean metric. 
Each surface considered in this paper will have boundary consisting of principal and asymptotic curves, which will allow us to determine the function $\zeta$. Once this is done, we can use (10) to conclude that

$$
d h=\frac{g(d \zeta)^{2}}{d g} .
$$

2.3. Extremal length The developing maps considered in this paper will turn out to be biholomorphic, edge-preserving maps between curvilinear polygons (polygons whose edges are arcs of circles or Euclidean line segments). Our proof of existence for these maps relies on the conformal invariant extremal length, some properties of which we now describe. For more details, see [Ahl73].

Given a curvilinear polygon $\Delta$ and a conformal metric $\rho\left(d x^{2}+d y^{2}\right)$ on $\Delta$, we denote the length of a curve $\gamma \subseteq \Delta$ with respect to this metric by $\ell \rho(\gamma)$. Similarly, we denote the $\rho$-area of a subset $U \subseteq \Delta$ by $A \rho(U)$. Using this notation, the extremal length between two connected subsets, $A$ and $B$, of $\partial \Delta$ is defined by

$$
\operatorname{Ext}_{\Delta}(A, B)=\sup _{\rho} \frac{\inf _{\gamma}[\ell \rho(\gamma)]^{2}}{A \rho(\Delta)},
$$

where the infimum is taken over all curves $\gamma:[0,1] \rightarrow \Delta$ such that $\gamma(0) \in A$, $\gamma(1) \in B$, and $\gamma(t) \subseteq$ interior $(\Delta)$ for $t \in(0,1)$; the supremum is taken over all positive Borel measurable functions on $\Delta$.

Furthermore, we define the symmetric measure between sets of Hausdorff dimension $k$ by $\mu^{k}\left[S_{1}, S_{2}\right]=\mathcal{H}^{k}\left[\left(S_{1} \cup S_{2}\right) \backslash\left(S_{1} \cap S_{2}\right)\right]$, and, given $A, B \subset \partial \Delta$ and $\tilde{A}, \tilde{B} \subset \partial \tilde{\Delta}$, we define the dilation distance by

$$
\operatorname{dila}[(\Delta, A, B),(\tilde{\Delta}, \tilde{A}, \tilde{B})]=\inf _{h}\left\{\mu^{2}(h(\Delta), \tilde{\Delta})+\mu^{1}(h(A), \tilde{A})+\mu^{1}(h(B), \tilde{B})\right\}
$$

where the infimum is taken over all compositions $h$ of dilations and translations.

Having noted these preliminaries, the properties of extremal length used below are as in the following result.

\section{PROPOSITION 3.}

(i) Extremal length is invariant under biholomorphisms.

(ii) Extremal length is continuous with respect to all three arguments in the sense that for any $\epsilon>0$, there is some $\delta$ such that $\operatorname{dila}[(\Delta, A, B),(\tilde{\Delta}, \tilde{A}, \tilde{B})]<\delta$ implies

$$
\left|\operatorname{Ext}_{\tilde{\Delta}}(\tilde{A}, \tilde{B})-\operatorname{Ext}_{\Delta}(A, B)\right|<\epsilon .
$$

(iii) If $A$ and $B$ are adjacent, that is, $\operatorname{dist}(A, B)=0$, then $\operatorname{Ext}_{\Delta}(A, B)=0$.

(iv) If $B$ is degenerate (that is, $B$ is a point) and $\operatorname{dist}(A, B)>0$, then

$$
\operatorname{Ext}_{\Delta}(A, B)=\infty
$$


(v) If $\Delta$ is a rectangle with edges $\left\{B_{k}\right\}, k=1,2,3,4$, such that $\left|B_{1}\right|=\left|B_{3}\right|=a$ and $\left|B_{2}\right|=\left|B_{4}\right|=b$, then

$$
\operatorname{Ext}_{\Delta}\left(B_{1}, B_{3}\right)=1 / \operatorname{Ext}_{\Delta}\left(B_{2}, B_{4}\right)=\frac{b}{a} .
$$

(vi) If $\Delta_{1} \subset \Delta_{2}$ are such that $A_{k}, B_{k} \subset \Delta_{k}, k=1,2$, satisfy $A_{1} \subset A_{2}$ and $B_{1} \subset B_{2}$, then

$$
\operatorname{Ext}_{\Delta_{2}}\left(A_{2}, B_{2}\right) \leq \operatorname{Ext}_{\Delta_{1}}\left(A_{1}, B_{1}\right),
$$

where the inequality is strict if $\operatorname{dist}\left(A_{2}, B_{2}\right)>0$ and either $A_{1} \neq A_{2}$ or $B_{1} \neq B_{2}$.

(vii) If $\Delta_{1} \subseteq \Delta_{2}$ are simply connected domains such that $A_{k}, B_{k} \subseteq \partial \Delta_{k}, k=1,2$, such that every path $\gamma$ connecting $A_{2}$ to $B_{2}$ must pass through $A_{1}$ and $B_{1}$, then

$$
\operatorname{Ext}_{\Delta_{1}}\left(A_{1}, B_{1}\right) \leq \operatorname{Ext}_{\Delta_{2}}\left(A_{2}, B_{2}\right) .
$$

\section{Construction of examples}

Our initial goal is to find, for each $\theta \in[0, \pi / 2)$, an annulus $\mathcal{A}=\mathcal{A}_{\theta}$ and a function $u=u_{\theta}$ defined on $\mathcal{A}$ such that $\operatorname{Graph}(u)$ is minimal and $u$ has a finite jump discontinuity at some point $p$ on the inner boundary component of the annulus. This last assertion means that

$$
-\infty<a:=\varliminf_{\mathcal{A} \ni q \rightarrow p} u(q)<b:=\varlimsup_{\mathcal{A} \ni q \rightarrow p} u(q)<\infty
$$

and that, for each $x \in[a, b]$, there is a sequence $\left\{p_{j}\right\} \in \mathcal{A}$ converging to $p$ such that

$$
\lim _{j \rightarrow \infty} u\left(p_{j}\right)=x
$$

We will achieve our goal by constructing a parametric minimal immersion in $\mathbb{R}^{3}$ that turns out to be a graph over an annular domain in the $x_{1} x_{2}$-plane. The jump discontinuity will be realized as a vertical line segment contained in the boundary of the surface and lying over a point on the inner boundary component of the annulus.

3.1. Basic geometry of the surface; proposed Gauss image We begin by assuming the existence of a minimal graph over some annulus centered at the origin and with a finite jump discontinuity located at the intersection of the inner boundary component with the positive $x_{1}$-axis. To simplify things, we assume that:

(S1) the graph is symmetric with respect to reflection through the $x_{2} x_{3}$-plane.

(Note that this adds a second jump discontinuity to the graph.) Additionally, we assume that the portion of the $x_{1}$-axis contained in the annulus is also contained in the graph. By the Schwarz reflection principle for minimal surfaces, it then follows that:

(S2) the graph is symmetric with respect to 180 degree rotation around the $x_{1}$-axis.

To describe the boundary of our graph, we partition the annulus boundary into the following four disjoint components: 

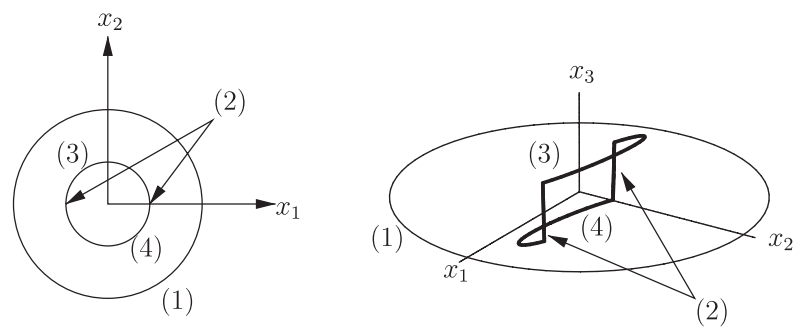

FIGURE 2. Boundary of annular domain and proposed boundary of minimal graph.

(1) the outer boundary component;

(2) the two points where the jump discontinuities occur;

(3) the (open) upper arc of the inner boundary component;

(4) the (open) lower arc of the inner boundary component.

Then we assume that the boundary of the graph consists of five curves: one over each of the components (1), (3) and (4), and two vertical line segments comprising the jump discontinuities (see Figure 2).

Additionally, we assume that the two curves over the upper and lower halves of the inner boundary component are tilted at an elevation angle of $\theta$, where $\theta \in[0, \pi / 2)$. More precisely, we assume that these curves lie in planes orthogonal to the vector $N_{\theta}=(0, \sin \theta,-\cos \theta)$. Then, so that these two curves will be principal curves, we assume that:

(GI) The Gauss map on the surface makes a constant angle of $\pi / 2$ with the vector $N_{\theta}$ along the boundary of the graph lying over the upper and lower halves of the inner boundary component.

The primary purpose of the curve over the outer boundary component, in this initial construction, is to complete the global graph. For simplicity, therefore, we assume that this curve lies in the horizontal plane $x_{3}=0$ and satisfies the following requirement:

(GO) The Gauss map on the surface makes a constant angle of $\phi \in(0, \pi / 2)$ with the positive $x_{3}$-axis along the outer boundary curve of the annulus, where

$$
R_{\phi}:=\frac{\sin \phi}{1+\cos \phi}<\frac{\cos \theta}{1+\sin \theta} .
$$

The seemingly mysterious inequality (12), as we will see below, ensures that the Gauss image of our surface makes sense. For the moment, we simply note that, given $\theta$, the condition (12) will always be satisfied for $\phi$ small enough.

In order to simplify the existence proof, we note that, from assumptions (S1) and (S2), it suffices to prove the existence of the portion of the graph lying over the first quadrant of the $x_{1} x_{2}$-plane. Such a fundamental piece is simply connected and bounded by a simple closed curve $\Gamma=\Gamma_{0} \cup \cdots \cup \Gamma_{4}$ (see Figure 3), where $\Gamma_{0}$ is a curve in the plane $x_{3}=0$ along which $(\mathrm{GO})$ holds, $\Gamma_{1}$ is a horizontal line segment 

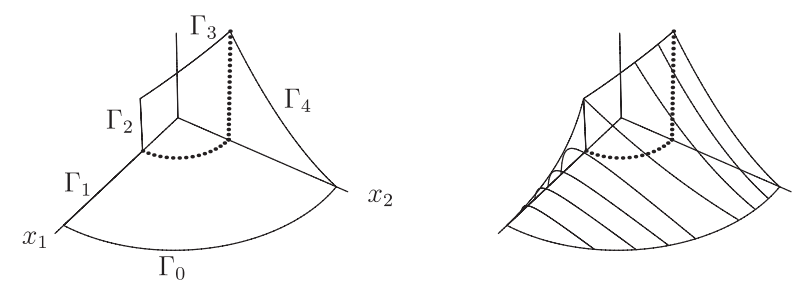

FIGURE 3 . The boundary and a sketch of the fundamental piece.

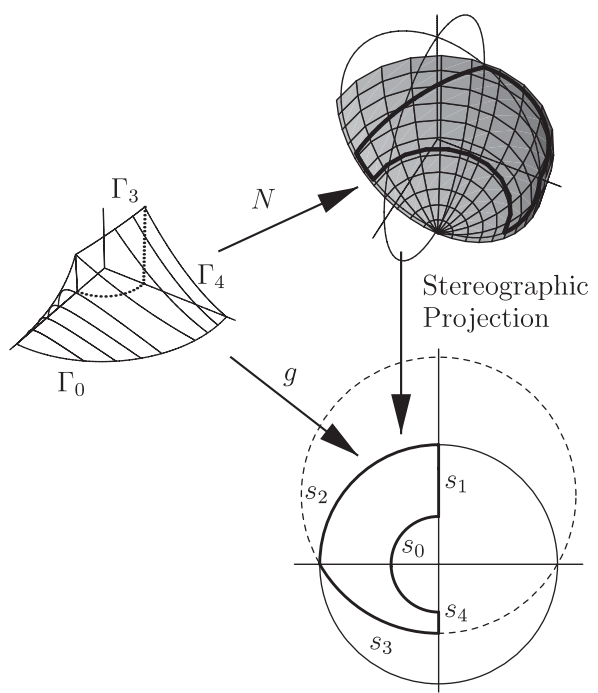

FIGURE 4. The Gauss image of the fundamental piece.

contained in the $x_{1}$-axis, $\Gamma_{2}$ is a vertical line segment, $\Gamma_{3}$ is a planar curve with elevation angle $\theta$ along which (GI) holds, and $\Gamma_{4}$ is a symmetry curve with respect to the $x_{2} x_{3}$-plane.

Under stereographic projection, the image of the Gauss map restricted to this boundary is shown in Figure 4. Here, $s_{0}$ is the half of $\partial B_{R \phi}(0)$ in the second and third quadrants, $s_{1}$ is the segment $\left[i R_{\phi}, i\right], s_{2}$ is the quarter of $\partial B_{1}(0)$ in the second quadrant, $s_{3}$ is the portion of $\partial B_{\sec \theta}(i \tan \theta)$ in the third quadrant, and $s_{4}$ is the segment $\left[-i(\sec \theta-\tan \theta),-i R_{\phi}\right]$. Since $\sec \theta-\tan \theta=\cos \theta /(1+\sin \theta)$, we see from the last segment $s_{4}$ that the mysterious condition (12) is precisely what is required for this image to bound a domain in $\mathbb{C}$. (Actually, equality in (12) also results in a well-defined domain; the resulting cusp, however, leads to integrability problems later which we wish to avoid.)

It is natural, moreover, to assume the Gauss image of the entire fundamental piece $\mathcal{G}_{0}$ is precisely the domain $\Omega=\Omega_{\theta} \subseteq \mathbb{C}$ just described and that the mapping $g: \mathcal{G}_{0} \rightarrow \Omega$ is one-to-one. 

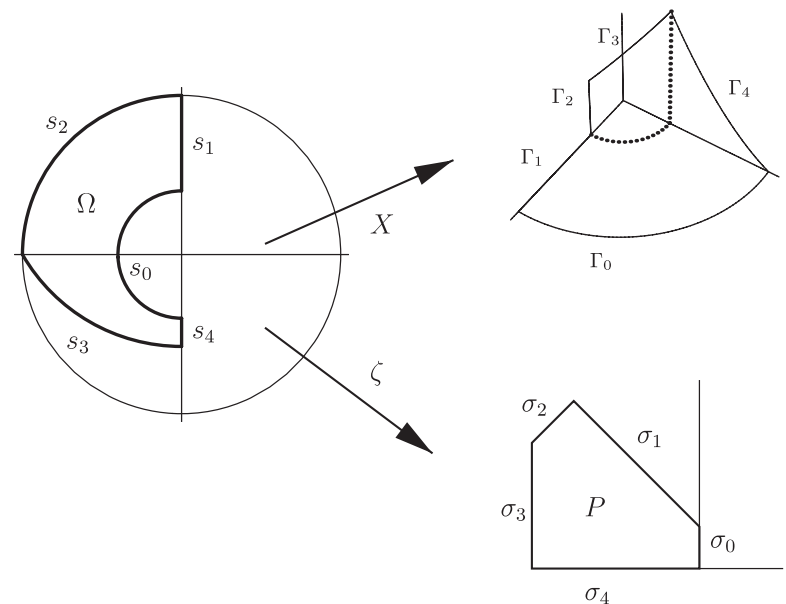

FIGURE 5. The Gauss image as parameter domain and the developing map.
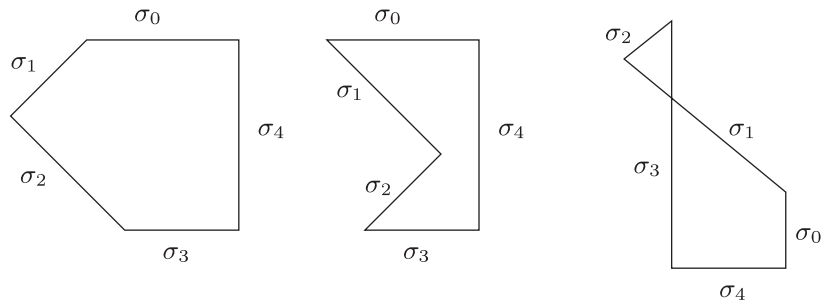

FIGURE 6. Other possible developing images.

At this point, we change our point of view and take the Gauss image $\Omega$ as a parameter domain for the surface. Thus, $X: \Omega \rightarrow \mathbb{R}^{3}$ defined by (5) gives the surface with $g: \Omega \rightarrow \mathbb{C}$ given by $g(z)=z$. It remains only to determine $d h$.

3.2. The developing map and height differential We seek now to determine the image of the developing map $\zeta=\zeta_{\theta, \phi}$ given by (10). To accomplish this, we first note that each curve $\Gamma_{j}$ is either an asymptotic curve or a principal curve. Indeed, it follows immediately that $\Gamma_{1}$ and $\Gamma_{2}$ are asymptotic. For $\Gamma_{0}, \Gamma_{3}$, and $\Gamma_{4}$, we have that each is a planar curve along which the surface meets the plane of the curve at a constant angle. By Joachimstahl's theorem, such curves are principal. Thus, by (9), the curves $\Gamma_{1}$ and $\Gamma_{2}$ are mapped by $\zeta$ into lines in one of the directions $e^{ \pm i \pi / 4}$, while $\Gamma_{0}, \Gamma_{3}$, and $\Gamma_{4}$ are mapped into horizontal or vertical lines. Based on this information, we conclude that the image of $\zeta$ is a Euclidean pentagon $P=P_{\theta}$ with edges oriented and labeled as in Figure 5.

There are, of course, other nominal possibilities for the image of $\zeta$ restricted to $\partial \Omega$; see Figure 6. Some of these can be used to obtain a piece of the surface we desire to 

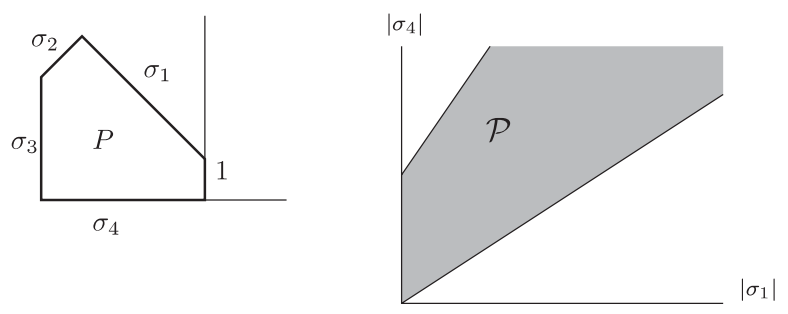

FIGURE 7. Parameter space for pentagonal developing images.

construct-but in a different orientation than suggested in Figure 5. Others produce different surfaces altogether. Each, and the properties of each, may be checked via calculations similar to those below. Nevertheless, it is clear that there are finitely many possibilities.

A certain amount of trial and error leads us to the image indicated in Figure 5. We wish, furthermore, to obtain an invertible edge-preserving conformal map $\zeta$ from the pentagon $\Omega$ to a pentagon of the shape $P$ indicated.

Normalizing so that $\left|\sigma_{0}\right|=1$, we note that each such pentagon is uniquely determined by the lengths $\left|\sigma_{1}\right|$ and $\left|\sigma_{4}\right|$. More precisely, the pentagons of interest are in one-to-one correspondence with

$$
\mathcal{P}=\left\{\left(\left|\sigma_{1}\right|,\left|\sigma_{4}\right|\right):\left|\sigma_{1}\right|>0 \text { and }\left|\sigma_{1}\right| / \sqrt{2}<\left|\sigma_{4}\right|<1+\left|\sigma_{1}\right| \sqrt{2}\right\}
$$

which we use to conveniently index this particular family of pentagons. The following existence result is crucial.

Proposition 4. For each $\theta \in[0, \pi / 2)$ and each $\phi$ small enough to satisfy (12), there exists a unique edge-preserving conformal map $\zeta=\zeta_{\theta, \phi}$ from $\Omega$ onto some pentagon $P$ corresponding to $\left(\left|\sigma_{1}\right|,\left|\sigma_{4}\right|\right) \in \mathcal{P}$.

PROOF. The parameter domain $\mathcal{P}$ is shown in Figure 7 , and restricting extremal length to any two sides of a pentagon corresponding to $\left(\left|\sigma_{1}\right|,\left|\sigma_{4}\right|\right) \in \mathcal{P}$, we obtain a function which is continuous on $\mathcal{P}$. For example, we may consider $\operatorname{Ext}_{\Delta}\left(\sigma_{2}, \sigma_{4}\right)$; since the entire pentagon (including $\left.\sigma_{2}\right)$ is determined by $\left(\left|\sigma_{1}\right|,\left|\sigma_{4}\right|\right) \in \mathcal{P}$, we may consider this as a function defined (and continuous) on $\mathcal{P}$. Let us slightly abuse notation by writing $P_{\sigma_{1}, \sigma_{4}} \in \mathcal{P}$ and temporarily denoting both side lengths and sides by $\sigma_{j}=\left|\sigma_{j}\right|$. We fix $\sigma_{1}$ and consider the 'segment of pentagons'

$$
\Delta=P_{\sigma_{1}, \sigma_{4}} \quad \text { with }\left|\sigma_{1}\right| / \sqrt{2}<\left|\sigma_{4}\right|<1+\left|\sigma_{1}\right| \sqrt{2}
$$

See Figure 8. By continuity and Proposition 3(iv), we see that

$$
\lim _{\left|\sigma_{4}\right| \searrow\left|\sigma_{1}\right| / \sqrt{2}} \operatorname{Ext}_{\Delta}\left(\sigma_{2}, \sigma_{4}\right)=\infty
$$



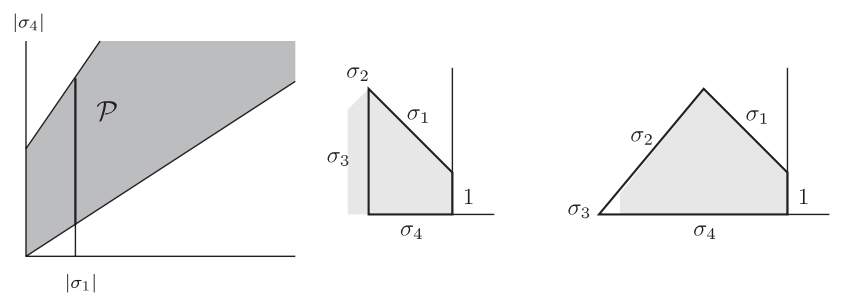

FIGURE 8. A segment in $\mathcal{P}$ with the lower and upper endpoint pentagons.

and by assertion (iii) of the same proposition,

$$
\lim _{\left|\sigma_{4}\right| \nearrow 1+\left|\sigma_{1}\right| / \sqrt{2}} \operatorname{Ext}_{\Delta}\left(\sigma_{2}, \sigma_{4}\right)=0 .
$$

It follows from the intermediate value theorem that there is some

$$
\left|\sigma_{4}^{*}\right| \in\left(\left|\sigma_{1}\right| / \sqrt{2}, 1+\left|\sigma_{1}\right| \sqrt{2}\right)
$$

such that

$$
\operatorname{Ext}_{\Delta}\left(\sigma_{2}, \sigma_{4}^{*}\right)=\operatorname{Ext}_{\Omega}\left(s_{2}, s_{4}\right)
$$

where $\Delta=P_{\sigma_{1}, \sigma_{4}^{*}}$. We have used here the fact that, since all the sides of $\Omega=\Omega_{\theta, \phi}$ have finite, nonzero length, the strict inequality of Proposition 3(vi) implies that all nonadjacent sides of the Gauss image $\Omega$ have finite, nonzero extremal length. We sharpen this last observation as follows.

Lemma 5. For $\sigma_{1}$ fixed and $\Delta=P_{\sigma_{1}, \sigma_{4}}$ as above, $\operatorname{Ext}_{\Delta}\left(\sigma_{2}, \sigma_{4}\right)$ is a decreasing function of $\left|\sigma_{4}\right|$. Consequently, $\sigma_{4}^{*}=\sigma_{4}^{*}\left(\left|\sigma_{1}\right|\right)$ is uniquely defined and continuous. Furthermore, $\sigma_{4}^{*}$ is increasing and $\lim _{\left|\sigma_{1}\right| \searrow 0} \sigma_{4}^{*}\left(\left|\sigma_{1}\right|\right)>0$.

PROOF. The monotonicity of $\operatorname{Ext}_{\Delta}\left(\sigma_{2}, \sigma_{4}\right)$ as a function of $\sigma_{4}$ follows immediately from Proposition 3(vi); see Figure 9 (left). Thus, $\sigma_{4}^{*}=\sigma_{4}^{*}\left(\left|\sigma_{1}\right|\right)$ is uniquely defined. The continuity follows from the continuity of extremal length, the monotonicity, and the defining condition (17). In fact, if we assume $\tilde{\sigma}_{4}=\lim \sup _{\tilde{\sigma}_{1} \rightarrow \sigma_{1}} \sigma_{4}^{*}\left(\tilde{\sigma}_{1}\right)>\sigma_{4}^{*}\left(\sigma_{1}\right)$, then $\left(\sigma_{1}, \tilde{\sigma}_{4}\right) \in \mathcal{P}$ (by continuity, $\tilde{\sigma}_{4}<1+\sigma_{1} \sqrt{2}$ ). By the monotonicity,

$$
\operatorname{Ext}_{\tilde{\Delta}}\left(\tilde{\sigma}_{2}, \tilde{\sigma}_{4}\right)<\operatorname{Ext}_{\Delta}\left(\sigma_{2}, \sigma_{4}^{*}\left(\sigma_{1}\right)\right)
$$

where $\tilde{\sigma}_{2}$ is the second side of $\tilde{\Delta}=P_{\sigma_{1}, \tilde{\sigma}_{4}}$. On the other hand, both sides of (18) are

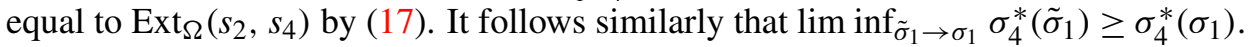

In order to see that $\sigma_{4}^{*}$ is increasing, assume the contrary, that is, assume that, for some $\left|\tilde{\sigma}_{1}\right|>\left|\sigma_{1}\right|$, we have $\sigma_{4}^{*}\left(\left|\tilde{\sigma}_{1}\right|\right) \leq \sigma_{4}^{*}\left(\left|\sigma_{1}\right|\right)$. We see then (see Figure 9 (middle)) that $\tilde{\Delta}:=P_{\tilde{\sigma}_{1}, \sigma_{4}^{*}\left(\left|\tilde{\sigma}_{1}\right|\right)}$ may be dilated (which does not change extremal length) so that the dilated pentagon has sides 2 and 4 strictly contained in the corresponding sides of $\Delta=P_{\sigma_{1}, \sigma_{4}^{*}\left(\left|\sigma_{1}\right|\right)}$. It follows from Proposition 3(vi) that

$$
\operatorname{Ext}_{\Omega}\left(s_{2}, s_{4}\right)=\operatorname{Ext}_{\tilde{\Delta}}\left(\tilde{\sigma}_{2}, \sigma_{4}^{*}\left(\left|\tilde{\sigma}_{2}\right|\right)>\operatorname{Ext}_{\Delta}\left(\sigma_{2}, \sigma_{4}^{*}\left(\left|\sigma_{1}\right|\right)\right)=\operatorname{Ext}_{\Omega}\left(s_{2}, s_{4}\right),\right.
$$

which is a contradiction to the above assumption. 

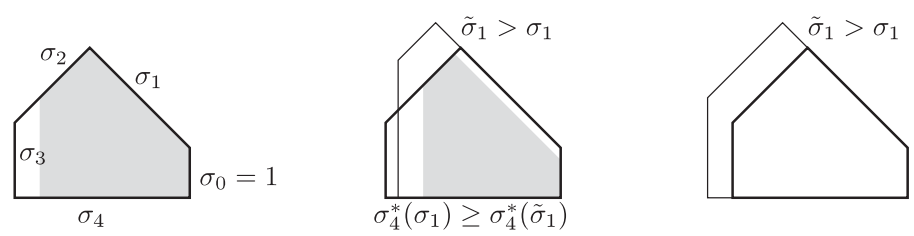

FIGURE 9. Proposition 3(vi) and the monotonicity of $\sigma_{4}^{*}$.

Finally, we consider values of $\sigma_{1}$ tending to zero and assume, by way of contradiction, that $\sigma_{4}^{*}\left(\sigma_{1}\right) \searrow 0$. Each corresponding pentagon must contain a rectangle having one side coincident with $\sigma_{4}^{*}$ and other side of length

$$
\min \left\{1,1+\sigma_{1} / 2-\sigma_{2} / \sqrt{2}\right\}>1-\sigma_{4}^{*} \quad\left(\text { since } \sigma_{4}^{*}>\sigma_{2} / \sqrt{2}\right) .
$$

Applying Proposition 3(vii) to this rectangle and $\Delta$, we find that

$$
\operatorname{Ext}_{\Omega}\left(s_{2}, s_{4}\right)=\operatorname{Ext}_{\Delta}\left(\sigma_{2}, \sigma_{4}\right) \geq \frac{\min \left\{1,1+\left(\sigma_{1}-\sigma_{2}\right) / \sqrt{2}\right\}}{\sigma_{4}^{*}}>\frac{1}{\sigma_{4}^{*}}-1 \rightarrow+\infty,
$$

which is a contraction. This completes the proof of Lemma 5.

Returning to the proof of Proposition 4, we consider $\operatorname{Ext}_{\Delta}\left(\sigma_{1}, \sigma_{4}\right)$ where $\Delta=P_{\sigma_{1}, \sigma_{4}^{*}\left(\sigma_{1}\right)}$ and $\sigma_{4}=\sigma_{4}^{*}\left(\sigma_{1}\right)$.

From Lemma 5 , we find that the pentagons $\Delta$ are nested with increasing $\sigma_{1}$ as indicated in Figure 9 (right). It follows, moreover, from Proposition 3 that $\operatorname{Ext}_{\Delta}\left(\sigma_{1}, \sigma_{4}\right)$ is decreasing in $\sigma_{1}$, and

$$
\lim _{\sigma_{1} \searrow 0} \operatorname{Ext}_{\Delta}\left(\sigma_{1}, \sigma_{4}\right)=+\infty
$$

(since $\sigma_{4}=\sigma_{4}^{*}$ remains bounded away from 0 ).

LEMMA 6. With $\sigma_{4}=\sigma_{4}^{*}\left(\sigma_{1}\right)$ as just described,

$$
\lim _{\sigma_{1} \nearrow \infty} \operatorname{Ext}_{\Delta}\left(\sigma_{1}, \sigma_{4}\right)=0
$$

PROOF. We know the limit exists by monotonicity. We can see the actual value by rescaling so that side $\tilde{\sigma}_{1}$ of the rescaled pentagon $\tilde{\Delta}$ has length 1 . Since

$$
\sigma_{1} / \sqrt{2} \leq \sigma_{4} \leq 1+\sigma_{1} \sqrt{2}
$$

we see that

$$
\tilde{\sigma}_{4} \leq 1 / \sigma_{1}+\sqrt{2} \rightarrow \sqrt{2}
$$

so $\tilde{\sigma}_{4}$ is bounded and may be assumed to converge to a finite nonzero limit-at least in a subsequence. The $\tilde{\sigma}_{0}$ side clearly has length zero in the limit and

$$
\operatorname{Ext}_{\Delta}\left(\sigma_{1}, \sigma_{4}\right)=\operatorname{Ext}_{\tilde{\Delta}}\left(\tilde{\sigma}_{1}, \tilde{\sigma}_{4}\right) \rightarrow 0
$$


This completes the proof of Lemma 6 and establishes that there is a unique pentagon $P \in \mathcal{P}$ for which

$$
\operatorname{Ext}_{P}\left(\sigma_{1}, \sigma_{4}\right)=\operatorname{Ext}_{\Omega}\left(s_{1}, s_{4}\right)
$$

and

$$
\operatorname{Ext}_{P}\left(\sigma_{2}, \sigma_{4}\right)=\operatorname{Ext}_{\Omega}\left(s_{2}, s_{4}\right)
$$

By the Riemann mapping theorem, there is a conformal map $\zeta$ from $\Omega$ onto $P$ with

$$
\zeta\left(v_{04}\right)=w_{04}, \quad \zeta\left(v_{34}\right)=w_{34} \quad \text { and } \quad \zeta\left(v_{23}\right)=w_{23}
$$

where $v_{i j}=s_{i} \cap s_{j}$ and $w_{i j}=\sigma_{i} \cap \sigma_{j}$. If we assume $\zeta\left(v_{12}\right) \in \operatorname{interior}\left(\sigma_{2}\right)$, then Proposition 3(vi) implies

$$
\operatorname{Ext}_{\Omega}\left(s_{2}, s_{4}\right)=\operatorname{Ext}_{\Delta}\left(\zeta\left(s_{2}\right), \sigma_{4}\right)>\operatorname{Ext}_{\Delta}\left(\sigma_{2}, \sigma_{4}\right)=\operatorname{Ext}_{\Omega}\left(s_{2}, s_{4}\right)
$$

by (25). If we assume $\zeta\left(v_{12}\right) \in \operatorname{interior}\left(\sigma_{1}\right) \cup \sigma_{0}$, then we see similarly that

$$
\operatorname{Ext}_{\Omega}\left(s_{2}, s_{4}\right)<\operatorname{Ext}_{\Delta}\left(\sigma_{2}, \sigma_{4}\right)=\operatorname{Ext}_{\Omega}\left(s_{2}, s_{4}\right) .
$$

Thus, we conclude that $\zeta\left(v_{12}\right)=w_{12}$. Knowing this, we may apply the same method to show that $\zeta\left(v_{01}\right)=w_{01}$. This completes the proof of Proposition 4 .

3.3. Understanding the image In view of Proposition 4, we may set

$$
d h=z\left(\frac{d \zeta}{d z}\right)^{2}=z \zeta^{\prime 2} d z
$$

for $z \in \Omega$ and consider the minimal immersion

$$
X(z)=\left(X_{(1)}, 0,0\right)+\operatorname{Re} \int_{v_{12}}^{z}\left(\frac{1}{2}\left(1-z^{2}\right), \frac{i}{2}\left(1+z^{2}\right), z\right)\left(\frac{d \zeta}{d z}\right)^{2}
$$

where $X_{(1)}>0$ is the $x_{1}$ coordinate of the proposed jump discontinuity (to be determined later).

The first observation we make is that

$$
X \text { is finite valued and continuous on } \bar{\Omega} \text {. }
$$

The only points at which this could fail are the vertices $v_{j k}=s_{j} \cap s_{k}$ of $\Omega$. An inspection of (26), moreover, indicates that the only way this assertion could fail is if boundary nonconformality (angle change) in the mapping $\zeta$ produces nonintegrability. More precisely, if $\phi_{j k}$ denotes the angle interior to $\Omega$ at $v_{j k}$ and $\psi_{j k}$ denotes the angle interior to $P$ at $w_{j k}=\sigma_{j} \cap \sigma_{k}$, then

$$
\zeta=\left(z-v_{j k}\right)^{\psi_{j k} / \phi_{j k}} \zeta_{0}(z)
$$

in a neighborhood of $v_{j k}$ where $\zeta_{0}$ is holomorphic and nonzero at $v_{j k}$. Thus,

$$
\zeta^{\prime 2}=\left(z-v_{j k}\right)^{2\left(\psi_{j k} / \phi_{j k}-1\right)} \tilde{\zeta}_{0}(z)
$$


where $\tilde{\zeta}_{0}$ is holomorphic and nonzero at $v_{j k}$. We see, in fact, that $\zeta$ is conformal at $v_{12}, v_{34}$ and $v_{04}$. At $v_{01}$ we find that $\psi_{01} / \phi_{01}=(3 \pi / 4) /(\pi / 2)=3 / 2$. Also, since $\phi_{23}=\pi-\theta<\pi, \psi_{23} / \phi_{23}=(3 \pi / 4) /(\pi-\theta)>3 / 4$. In every case, $\psi_{j k} / \phi_{j k}>1 / 2$ so that $2\left(\psi_{j k} / \phi_{j k}-1\right)>-1$ and $\zeta^{\prime 2}$ is integrable. This proves (27).

We will need somewhat more precise information near $v_{23}$ later, and we take this opportunity to note that in that case (28) becomes

$$
\zeta^{\prime 2}=\left(z-v_{23}\right)^{4 \theta-\pi / 2(\pi-\theta)} \tilde{\zeta}_{0}(z)
$$

From this we see that:

(Z1) $\zeta^{\prime}\left(v_{23}\right)^{2}=\infty$ for $0 \leq \theta<\pi / 4$;

(Z2) $\zeta^{\prime}\left(v_{23}\right)^{2}$ is finite and nonzero for $\theta=\pi / 4$; and

(Z3) $\zeta^{\prime}\left(v_{23}\right)^{2}=0$ for $\pi / 4<\theta<\pi / 2$.

These observations will be used to verify the assertions of Theorem 1 concerning the curvature of the inner boundary component.

Since, up to a nonvanishing conformal factor, the image $X$ is obtained by integrating $\zeta^{\prime 2} d z$, we may also determine from (28) the image angles $\alpha_{j k}$ interior to the immersed minimal image at the boundary points $X_{j k}=X\left(v_{j k}\right)$. We find that

$$
\begin{aligned}
& \alpha_{12}=\alpha_{34}=\alpha_{04}=\pi / 2, \\
& \alpha_{01}=\frac{\pi}{2}\left[2\left(\frac{3}{2}-1\right)+1\right]=\pi \text { and } \\
& \alpha_{23}=(\pi-\theta)\left[\frac{4 \theta-\pi}{2(\pi-\theta)}+1\right]=\theta+\pi / 2 .
\end{aligned}
$$

Each of these angles, with the exception of $\alpha_{01}$, might have been anticipated from Figures 2, 4 or 5 and the attendant discussion. We will find, contrary to what was anticipated, that the outer boundary curve will resemble the curve in Figure 10. In order to obtain a smooth annular domain as described in Theorem 1, we need only take as outer boundary a convex curve lying between the inner and outer boundary curves initially obtained.

We now proceed to verify the anticipated features of the image surface in Figure 4 (in light of this minor modification). We may parameterize $s_{1}$ from $v_{12}$ to $v_{01}$ by

$$
z_{1}(t)=i(1-t), \quad 0 \leq t \leq R_{\phi}
$$

Then we have $d z\left(\dot{z}_{1}\right)=-i$ and $d \zeta\left(\dot{z}_{1}\right)^{2}=-i\left|d \zeta\left(\dot{z}_{1}\right)\right|^{2}$. Thus,

$$
\begin{aligned}
& d X_{1}\left(\dot{z}_{1}\right)=\operatorname{Re}\left[\frac{1}{2}\left(1-z_{1}^{2}\right) \frac{d \zeta\left(\dot{z}_{1}\right)^{2}}{d z\left(\dot{z}_{1}\right)}\right]=\frac{\left|d \zeta\left(\dot{z}_{1}\right)\right|^{2}}{2}\left[1+(1-t)^{2}\right]>0 \\
& d X_{2}\left(\dot{z}_{1}\right)=\operatorname{Re}\left[\frac{i}{2}\left(1+z_{1}^{2}\right)\left|d \zeta\left(\dot{z}_{1}\right)\right|^{2}\right]=0 \quad \text { and } \\
& d X_{3}\left(\dot{z}_{1}\right)=\operatorname{Re}\left[z_{1}\left|d \zeta\left(\dot{z}_{1}\right)\right|^{2}\right]=0 .
\end{aligned}
$$




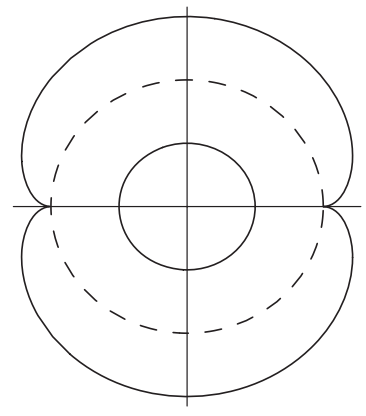

FIGURE 10. The initial annular domain and an alternative inner boundary.

These calculations imply that the image of $s_{1}$ is a horizontal line segment on the positive $x_{1}$-axis (as desired).

Next, we parameterize $s_{2}$ by

$$
z_{2}(t)=e^{i t}, \quad \pi / 2 \leq t \leq \pi .
$$

Then $d z\left(\dot{z}_{2}\right)=i e^{i t}$ and $d \zeta\left(\dot{z}_{2}\right)^{2}=i\left|d \zeta\left(\dot{z}_{2}\right)\right|^{2}$, so that

$$
\begin{aligned}
d X_{1}\left(\dot{z}_{2}\right) & =\operatorname{Re}\left[\frac{1}{2}\left(1-e^{2 i t}\right)\left|d \zeta\left(\dot{z}_{2}\right)\right|^{2} / e^{i t}\right] \\
& =\operatorname{Re}\left[-i \sin t\left|d \zeta\left(\dot{z}_{2}\right)\right|^{2}\right]=0, \\
d X_{2}\left(\dot{z}_{2}\right) & =\operatorname{Re}\left[\frac{i}{2}\left(1+e^{2 i t}\right)\left|d \zeta\left(\dot{z}_{2}\right)\right|^{2} / e^{i t}\right] \\
& =\operatorname{Re}\left[-i \cos t\left|d \zeta\left(\dot{z}_{2}\right)\right|^{2}\right]=0 \quad \text { and } \\
d X_{3}\left(\dot{z}_{2}\right) & =\operatorname{Re}\left[e^{i t}\left|d \zeta\left(\dot{z}_{2}\right)\right|^{2} / e^{i t}\right]=\left|d \zeta\left(\dot{z}_{2}\right)\right|^{2}>0 .
\end{aligned}
$$

This shows that $\Gamma_{2}$ is a vertical segment.

For $s_{3}$,

$$
\begin{gathered}
z_{3}(t)=i \tan \theta+\sec \theta e^{i t}, \quad \pi+\theta \leq t \leq 3 \pi / 2 \\
d z\left(\dot{z}_{3}\right)=i \sec \theta e^{i t} \quad \text { and } \quad d \zeta\left(\dot{z}_{3}\right)^{2}=-\left|d \zeta\left(\dot{z}_{3}\right)\right|^{2}<0 .
\end{gathered}
$$

Thus, we find that

$$
\begin{aligned}
d X_{1}\left(\dot{z}_{3}\right) & =\operatorname{Re}\left[\frac{1}{2}\left(1+\tan ^{2} \theta-2 i \tan \theta \sec \theta e^{i t}-\sec ^{2} \theta e^{z i t}\right) \frac{d \zeta\left(\dot{z}_{3}\right)^{2}}{\left(i \sec \theta e^{i t}\right)}\right] \\
& =-\frac{d \zeta\left(\dot{z}_{3}\right)^{2}}{2} \operatorname{Re}\left[i\left(\sec \theta e^{-i t}-2 i \tan \theta-\sec \theta e^{i t}\right)\right] \\
& =-d \zeta\left(\dot{z}_{3}\right)^{2} \operatorname{Re}[\sec \theta \sin t+\tan \theta] \\
& =-\frac{d \zeta\left(\dot{z}_{3}\right)^{2}}{\cos \theta}(\sin t+\sin \theta) \leq 0
\end{aligned}
$$


with strict inequality for $\pi+\theta<t<3 \pi / 2$ since $\sin t<\sin (\pi+\theta)=-\sin \theta$ on this interval. Also,

$$
\begin{aligned}
d X_{2}\left(\dot{z}_{3}\right) & =\frac{d \zeta\left(\dot{z}_{3}\right)^{2}}{2 \sec \theta} \operatorname{Re}\left[\left(1-\tan ^{2} \theta\right) e^{-i t}+2 i \tan \theta \sec \theta+\sec ^{2} \theta e^{i t}\right] \\
& =\frac{d \zeta\left(\dot{z}_{3}\right)^{2}}{2 \sec \theta}\left(1-\tan ^{2} \theta+\sec ^{2} \theta\right) \cos t \\
& =d \zeta\left(\dot{z}_{3}\right)^{2} \cos \theta \cos t \geq 0
\end{aligned}
$$

with strict inequality unless $t=3 \pi / 2$, and

$$
\begin{aligned}
d X_{3}\left(\dot{z}_{3}\right) & =\frac{d \zeta\left(\dot{z}_{3}\right)^{2}}{\sec \theta} \operatorname{Re}\left[\tan \theta e^{-i t}-i \sec \theta\right] \\
& =d \zeta\left(\dot{z}_{3}\right)^{2} \sin \theta \cos t \geq 0
\end{aligned}
$$

again with strict inequality unless $t=3 \pi / 2$.

To see that $\Gamma_{3}$ lies in a plane orthogonal to $N_{\theta}=(0, \sin \theta,-\cos \theta)$, we compute

$$
N_{\theta} \cdot d X\left(\dot{z}_{3}\right)=\sin \theta d \zeta\left(\dot{z}_{3}\right)^{2} \cos \theta \cos t-\cos \theta d \zeta\left(\dot{z}_{3}\right)^{2} \sin \theta \cos t=0 .
$$

Furthermore,

$$
\frac{d X_{1}\left(\dot{z}_{3}\right)}{d X_{2}\left(\dot{z}_{3}\right)}=-\frac{1}{\cos ^{2} \theta}(\sin t+\sin \theta) \sec t
$$

which is negative and decreasing in $t$ with $d X_{1} / d X_{2}=0$ at $t=\pi+\theta$ and $d X_{2} / d X_{1}=0$ at $t=3 \pi / 2$. This shows that $\Gamma_{3}$ projects (simply) onto one fourth of a convex $C^{1}$ curve in the $x_{1} x_{2}$-plane. If we now select

$$
X_{(1)}=-\operatorname{Re} \int_{s_{3}} \frac{1}{2}\left(1-z^{2}\right) \zeta^{\prime 2} d z>0,
$$

then the projection of $X\left(v_{14}\right)$ will lie on the positive $x_{2}$-axis and this convex $C^{1}$ curve may be assumed symmetric with respect to the $x_{1}$-axis and the $x_{2}$-axis. We have thus verified the basic assertions concerning $\Gamma_{3}$ and the inner boundary component of the annulus. (The question of curvature will be taken up in the next section.)

We next parameterize $s_{4}$, and hence $\Gamma_{4}$ :

$$
z_{4}(t)=i t, \quad \tan \theta-\sec \theta<t<-R_{\phi} .
$$

Thus,

$$
\begin{aligned}
d z\left(\dot{z}_{4}\right) & =i, \quad d z\left(\dot{z}_{4}\right)^{2}>0 \\
d X_{1}\left(\dot{z}_{4}\right) & =\frac{d \zeta\left(\dot{z}_{4}\right)^{2}}{2} \operatorname{Re}\left[\left(1+t^{2}\right) / i\right]=0 \\
d X_{2}\left(\dot{z}_{4}\right) & =\frac{d \zeta\left(\dot{z}_{4}\right)^{2}}{2} \operatorname{Re}\left[1-t^{2}\right]>0 \quad \text { and } \\
d X_{3}\left(\dot{z}_{4}\right) & =\frac{d \zeta\left(\dot{z}_{4}\right)^{2}}{2} t<0
\end{aligned}
$$


Thus, $\Gamma_{4}$ has the properties prescribed in Section 2.1. We also see that

$$
\frac{d X_{3}\left(\dot{z}_{4}\right)}{d X_{2}\left(\dot{z}_{4}\right)}=\frac{t}{1-t^{2}}
$$

and

$$
\frac{d}{d t}\left(\frac{t}{1-t^{2}}\right)=\frac{1}{1-t^{2}}+\frac{2 t^{2}}{\left(1-t^{2}\right)^{2}}=\frac{1+t^{2}}{1-t^{2}}>0,
$$

so that $\Gamma_{4}$ is convex when considered as a graph over the positive $x_{2}$-axis. The actual final height

$$
\begin{aligned}
X_{3}\left(v_{04}\right)= & \operatorname{Re} \int_{s_{2} \cup s_{3} \cup s_{4}} z \zeta^{\prime 2} d z \\
= & \int_{\pi / 2}^{\pi}\left|d \zeta\left(\dot{z}_{2}\right)\right|^{2} d t-\int_{\pi+\theta}^{3 \pi / 2} \sin \theta \cos t\left|d \zeta\left(\dot{z}_{3}\right)\right|^{2} d t \\
& \quad+\frac{1}{2} \int_{\tan \theta-\sec \theta}^{-R_{\phi}} t\left|d \zeta\left(\dot{z}_{4}\right)\right|^{2} d t
\end{aligned}
$$

is not clear at this point. We know by continuity, however, that

$$
X_{3}\left(v_{04}\right)=\operatorname{Re} \int_{-s_{0}} z \zeta^{\prime 2} d z
$$

where the integration along $-s_{0}$ indicates the clockwise direction with respect to $\Omega$. This, and the other basic properties of $\Gamma_{0}$, we now compute. Set

$$
z_{0}(t)=R_{\phi} e^{i t}, \quad \pi / 2 \leq t \leq 3 \pi / 2 .
$$

Then $d z\left(\dot{z}_{0}\right)=i R_{\phi} e^{i t}$ and $d \zeta\left(\dot{z}_{0}\right)^{2}=-\left|d \zeta\left(\dot{z}_{0}\right)\right|^{2}$, and so

$$
\begin{aligned}
d X_{1}\left(\dot{z}_{0}\right) & =\frac{\left|d \zeta\left(\dot{z}_{0}\right)\right|^{2}}{2 R_{\phi}} \operatorname{Re}\left[i\left(1-R_{\phi}^{2} e^{2 i t}\right) / e^{i t}\right] \\
& =\frac{\left|d \zeta\left(\dot{z}_{0}\right)^{2}\right|}{2 R_{\phi}}\left(1+R_{\phi}^{2}\right) \sin t \\
d X_{2}\left(\dot{z}_{0}\right) & =-\frac{\left|d \zeta\left(\dot{z}_{0}\right)\right|^{2}}{2 R_{\phi}} \operatorname{Re}\left[\left(e^{-i t}+R_{\phi}^{2} e^{i t}\right)\right] \\
& =-\frac{\left|d \zeta\left(\dot{z}_{0}\right)\right|^{2}}{2 R_{\phi}}\left(1+R_{\phi}^{2}\right) \cos t \geq 0
\end{aligned}
$$

with strict inequality away from the endpoints. Also,

$$
d X_{3}\left(\dot{z}_{0}\right)=\operatorname{Re}\left[i\left|d \zeta\left(\dot{z}_{0}\right)\right|^{2}\right]=0 .
$$


Thus, we see that $x_{3}\left(v_{04}\right)=0$. Furthermore, $\Gamma_{0}$ is concave down as a graph over the $x_{2}$-axis since

$$
\frac{d X_{1}\left(\dot{z}_{0}\right)}{d X_{2}\left(\dot{z}_{0}\right)}=-\tan t
$$

decreases from $+\infty$ to $-\infty$ with $t \in(\pi / 2,3 \pi / 2)$.

We have shown that the boundary of $X(\Omega)$ determines two initial boundary curves as indicated in Figure 10. Since the image of the Gauss map, $\Omega$, has interior in the unit disk, we see that $X(\Omega)$ is an embedded graph over the resulting projection in the first quadrant. Furthermore, $X(\bar{\Omega})$ may be extended by reflection in the $x_{2} x_{3}$-plane and 180 degree rotation about the $x_{1}$-axis to yield a graph of a function $u=u_{\theta}$ over the entire annulus. Clearly $u_{\theta}$ has jump discontinuities on the inner boundary curve at $\left( \pm X_{(1)}, 0\right)$. We next investigate the curvature of $\partial A$ at these points.

3.4. Curvature calculations Consider the projection $C$ of $\Gamma_{3}$ into the $x_{1} x_{2}$-plane. This is one fourth of the inner boundary curve of the annulus $A$, and we wish to compute the curvature of $C$ at the point $p=\left(X_{1}\left(v_{23}\right), X_{2}\left(v_{23}\right)\right)=\left(X_{1}\left(v_{12}\right), X_{2}\left(v_{12}\right)\right)$ where the jump discontinuity occurs.

Since $C$ is the graph of some function $f$ over an interval of the $x_{2}$-axis, we can write

$$
X_{1}=f\left(X_{2}\right)
$$

where $\left(X_{1}, X_{2}\right)=\left(X_{1}\left(z_{3}(t)\right), X_{2}\left(z_{3}(t)\right)\right)$ and the parameterization $z_{3}=z_{3}(t)$ of $s_{3}$ is given in (29) above. The curvature $\kappa$ at $p$ is found by evaluating

$$
\kappa(t)=\frac{f^{\prime \prime}}{\left(1+f^{\prime 2}\right)^{3 / 2}}
$$

at $t=\pi+\theta$. We recall from (30) that

$$
f^{\prime}=-\sec ^{2} \theta \sec t(\sin t+\sin \theta)
$$

We may also compute

$$
\begin{aligned}
f^{\prime \prime} & =\frac{d}{d t}\left[-\sec ^{2} \theta \sec t(\sin t+\sin \theta)\right] \frac{1}{d X_{2}\left(\dot{z}_{3}\right)} \\
& =-\frac{\sec ^{2} \theta[\sec t \tan t(\sin t+\sin \theta)+1]}{\cos \theta \sec t d \zeta\left(\dot{z}_{3}\right)^{2}} \\
& =-\frac{\sec ^{2} \theta \sec ^{2} t(1+\sin \theta \sin t)}{\cos \theta \cos t d \zeta\left(\dot{z}_{3}\right)^{2}} \\
& =-\frac{\sec ^{3} \theta \sec ^{3} t(1+\sin \theta \sin t)}{d \zeta\left(\dot{z}_{3}\right)^{2}}<0 .
\end{aligned}
$$




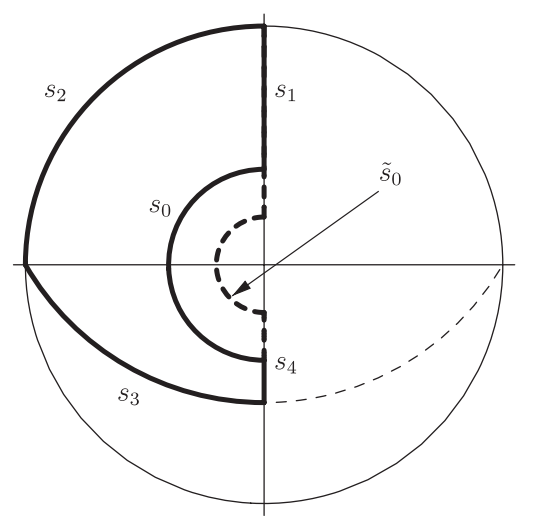

FIGURE 11. Dependence of the Gauss image on the outer angle parameter $\phi$.

In particular, evaluating at $t=\pi+\theta$,

$$
\kappa(p)=f^{\prime \prime}(0)=\frac{\sec ^{4} \theta}{d \zeta\left(\dot{z}_{3}(\pi+\theta)\right)^{2}}<0 .
$$

Since $d \zeta^{2}=\zeta^{\prime 2} d z$, we see from (Z1), (Z2), (Z3) of Section 3.3 that:

(K1) $\kappa(p)=0$ for $0 \leq \theta<\pi / 4$;

(K2) $-\infty<\kappa(p)<0$ for $\theta=\pi / 4$; and

(K3) $\kappa(p)=-\infty$ for $\pi / 4<\theta<\pi / 2$.

This completes the proof of Theorem 1.

3.5. Extensions and generalizations We consider here the limit as $\phi \searrow 0$ of the surfaces constructed above. For $\tilde{\phi}<\phi$, the Gauss image changes as indicated in Figure 11. Let us denote these Gauss images by $\Omega$ and $\tilde{\Omega}$. Next, we scale the pentagons associated with $\phi$ and $\tilde{\phi}$ from Proposition 4 so that the $\sigma_{4}$-sides have unit length and we denote these pentagons by $P$ and $\tilde{P}$. We claim that the pentagon $\tilde{P}$ associated with the smaller value $\tilde{\phi}$ must be nested inside the pentagon $P$ associated with $\phi$ as indicated in Figure 12 (left). The remaining possibilities are shown also in Figure 12; in each case $\tilde{P}$ is shaded. We know, however, from the monotonicity of extremal length, that

$$
\operatorname{Ext}_{\tilde{\Omega}}\left(s_{2}, \tilde{s}_{4}\right)<\operatorname{Ext}_{\Omega}\left(s_{2}, s_{4}\right)
$$

and

$$
\operatorname{Ext}_{\tilde{\Omega}}\left(\tilde{s}_{1}, \tilde{s}_{4}\right)<\operatorname{Ext}_{\Omega}\left(s_{1}, s_{4}\right) .
$$

Thus, in the second possibility, we can shrink $\tilde{P}$ further so that the $\tilde{\sigma}_{2}$-side falls into $\sigma_{2}$, and we find a contradiction of (32). In the third possibility, we can shrink $\tilde{P}$ so that the $\tilde{\sigma}_{2}$-side falls into $\sigma_{1}$ and (33) is contradicted. At least one of these arguments rules out the fourth possibility, and our claim is established. 

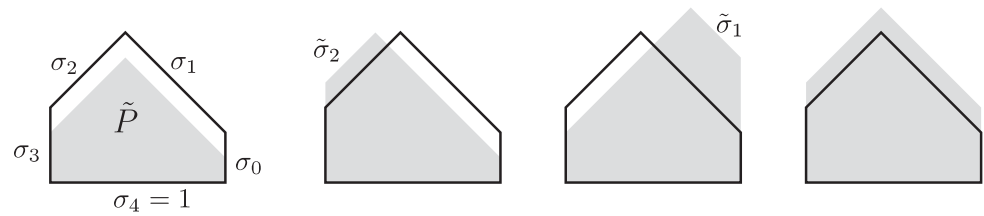

FIGURE 12. The associated pentagons.

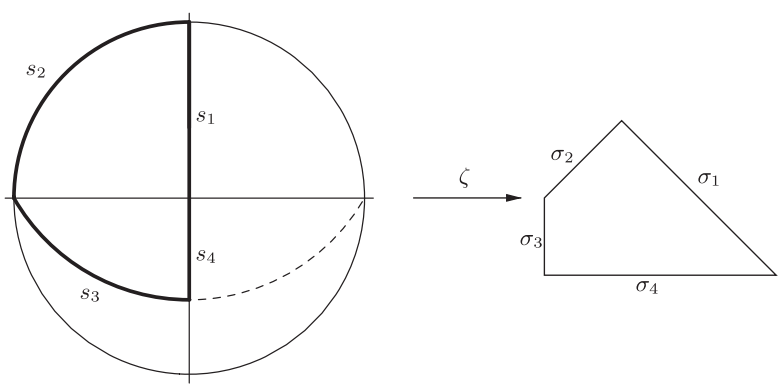

FIGURE 13. The developing map for the entire graph.

Thus, using the continuity of extremal length, we find that the limit as $\phi \searrow 0$ corresponds to a well-defined quadrilateral $Q$ with extremal lengths corresponding to the curvilinear quadrilateral $\Omega_{0}$ obtained as the limit of $\Omega_{\theta, \phi}$ as $\phi \searrow 0$; see Figure 13 .

The Riemann mapping $\zeta_{0}$ with $\zeta_{0}\left(v_{j k}\right)=w_{j k}$ for $j=1,2,3$ and $k=j+1$ is easily seen to satisfy also $\zeta_{0}\left(v_{24}\right)=w_{14}$. The resulting immersion $X$, however, does not extend continuously to $\bar{\Omega}_{0}$ since at the origin

$$
\zeta=z^{(\pi / 4) / \pi} \zeta_{1}=z^{1 / 4} \zeta_{1},
$$

where $\zeta_{1}$ is conformal at $z=0$, and

$$
\zeta^{\prime 2}=z^{-3 / 2} \tilde{\zeta}_{1}
$$

is not integrable at $z=0$. We thus obtain a minimal graph $u_{0}$ over the domain $A$ exterior to a convex domain whose boundary has curvature dependent on $\theta$ as described by (A), (K1), (K2) and (K3). This is the content of Theorem 2.

We remark, finally, that certain values of an additional angle $\psi$ can be introduced into the basic construction, so that the condition on the Gauss map restricted to the outer boundary $(\mathrm{GO})$ is replaced with the condition that the outer boundary component lies in the plane through the origin orthogonal to the vector $N_{\psi}=(0, \sin \psi,-\cos \psi)$ and the angle between the Gauss map restricted to this outer boundary component with $N_{\psi}$ is $\phi$. In this way, we can generalize the outer boundary condition along the lines used in the construction of the inner boundary. Since this last observation is not obviously of enough immediate interest to justify recording the somewhat complicated details associated with it, we omit them. 


\section{References}

[Ahl73] L. Ahlfors, Conformal Invariants: Topics in Geometric Function Theory (McGraw-Hill, New York, 1973).

[ET99] I. Ekeland and R. Témam, Convex Analysis and Variational Problems (SIAM, Philadelphia, PA, 1999).

[Fin63] R. Finn, 'New estimates for equations of minimal surface type', Arch. Ration. Mech. Anal. 14 (1963), 337-375.

[Giu84] E. Giusti, Minimal Surfaces and Functions of Bounded Variation (Birkhaüser, Boston, MA, 1984).

[HK97] D. Hoffman and H. Karcher, 'Complete embedded minimal surfaces of finite total curvature', in: Encyclopedia of Mathematics (ed. R. Osserman) (Springer, Berlin, 1997), pp. 5-93.

[JS68] H. Jenkins and J. Serrin, 'The Dirichlet problem for the minimal surface equation in higher dimensions', J. Reine Angew. Math. 229 (1968), 170-187.

[Lan06] K. Lancaster, 'A proof of the Concus-Finn conjecture', Preprint, 2006.

[Mir71] M. Miranda, 'Un principio di massimo forte per le frontiere minimali e una sua applicazione alla risoluzione del problema al contorno per l'equazione delle superfici di area minima', Rend. Sem. Mat. Univ. Padova 45 (1971), 355-366.

[Sim74] L. Simon, 'Global estimates of Hölder continuity for a class of divergence-form elliptic equations', Arch. Ration. Mech. Anal. 56 (1974), 253-272.

[Sim82] , 'Boundary behavior of solutions of the nonparametric least area problem', Bull. Austral. Math. Soc. 26 (1982), 17-27.

[Wil84] G. Williams, 'The Dirichlet problem for the minimal surface equation with Lipschitz continuous boundary data', J. Reine Angew. Math. 354 (1984), 123-140.

[Wil86a] _ ' Global regularity for solutions of the minimal surface equation with continuous boundary values', Ann. Inst. H. Poincaré (C) Anal. Non Linéaire 3(6) (1986), 411-429.

[Wil86b] - 'Solutions of the minimal surface equation-continuous and discontinuous at the boundary', Comm. Partial Differential Equations 11 (1986), 1439-1457.

[Wil87] _ 'The best modulus of continuity for solutions of the minimal surface equation', Pacific J. Math. 129 (1987), 193-208.

ROBERT HUFF, Department of Mathematical Sciences, Indiana University,

South Bend, IN 46634, USA

e-mail: rohuff@iusb.edu

JOHN MCCUAN, Department of Mathematics, Georgia Institute of Technology, Atlanta, GA 30332, USA

e-mail: mccuan@math.gatech.edu 